\title{
CONTINUOUS SELECTIONS WITH NONMETRIZABLE RANGE
}

\author{
BY \\ H. H. CORSON(1) AND J. LINDENSTRAUSS(2)
}

1. Introduction. Let $X$ and $L$ be topological spaces and let $\phi$ be a map from $X$ to $2^{L}$ the space of all nonempty subsets of $L$. A continuous function $f$ from $X$ to $L$ is said to be a continuous selection for $\phi$ if $f(x) \in \phi(x)$ for every $x \in X$. The notion of continuous selection was introduced and studied in detail by E. Michael (cf. [10], [11], [12] and the references there). We shall consider here only mappings $\phi$ which are lower semicontinuous (1.s.c.), that is, mappings for which $\{x: \phi(x) \cap V \neq \varnothing\}$ is an open subset of $X$ for every open subset $V$ of $L$. This is a natural restriction on $\phi$ since, as observed in [10], it is a necessary condition for the existence of selections for $\phi$ which take at a given point $x_{0} \in X$ a preassigned value $y_{0}$ in $\phi\left(x_{0}\right)$. If $L$ is metrizable (or even only first countable) and $X$ is the one point compactification of the integers, then it is easily seen that every 1.s.c. map $\phi$ from $X$ to $2^{L}$ admits a continuous selection. It is also easily seen that this is no longer the case if $L$ is not first countable. This simple observation is the main reason for the difficulties encountered in proving and applying selection theorems with a nonmetrizable range.

Selection theorems are in an obvious way generalizations of extension theorems. An extension problem is a selection problem in which $\phi(x)$ is, for every $x \in X$, either a single point or the whole space $L$. In the introduction of [10] Michael pointed out that the main result of his selection theory is the fact that most of the known extension theorems can be slightly changed and essentially generalized to suitable selection theorems. The known extension theorems for functions from a topological space $X$ to a topological linear space $L$ fall into two classes. One class of these theorems states that under various normality conditions on $X$ every continuous function from a closed subset $X_{0}$ of $X$ into a metrizable locally convex space $L$ (or only into a Banach or a separable Banach space) can be extended to a continuous function from $X$ to $L$. The paper [10] is devoted to the proofs and applications of selection theorems which correspond to these extension theorems.

In the second class of extension theorems the main assumption is the metrizability of the domain $X$ rather than the range $L$. The main result in this direction,

Presented to the Society, April 24, 1965; received by the editors April 23, 1965.

(1) Research fellow of the Alfred P. Sloan Foundation.

(2) Research supported in part by the National Science Foundation (NSF-GP-378). 
due to Dugundji [5], states that if $X$ is metrizable and if $X_{0}$ is a closed subset of $X$, then every continuous function $f$ from $X_{0}$ to a locally convex space $L$ can be extended to a continuous function $F$ from $X$ to $L$ so that $F(X)$ is contained in the convex hull of $f\left(X_{0}\right)$. The purpose of this paper is to prove selection theorems which correspond to this extension theorem.

The main question which arises in this connection is the following. For which metric spaces $X$ and linear spaces (or subsets of linear spaces) $L$, is it true that every 1.s.c. map $\phi$ from $X$ to $2^{L}$, for which $\phi(x)$ is closed and convex for every $x \in X$, admits a continuous selection? The assumption that $\phi(x)$ is closed and convex for every $x$ is a natural one (it is through the convexity assumption that the linear structure of $L$ enters into the selection problem) and, as Michael showed in [10], it is also an unavoidable assumption.

It seems unlikely, in view of the many different situations which arise, that it is possible to characterize in a reasonable manner all pairs of $X$ and $L$ for which the above selection theorem is valid. The results we prove in this paper (together with the examples) give, however, a reasonably good understanding of the general situation. It turns out that the selection theorem holds for nonmetrizable linear spaces $L$ only if their topology is sufficiently weak. For example, if we take a Banach space in the weak $(w)$ topology then in general no continuous selection exists but it is often possible to take a still weaker topology than the $w$-topology and thereby obtain a Hausdorff locally convex space for which the selection theorem holds (if $X$ is separable and complete). Thus in order to obtain selection theorems for the more common linear topological spaces one has to restrict the attention to topological spaces $L$ which are compact sets in linear spaces rather than whole linear spaces. Examples show also that the selection theorem may fail if $L$ is a general compact set in a locally convex space. The right setting for the selection theorems seems to be the situation where $L$ is a $w$-compact set in a Banach space.

We state now a special case of our selection theorem which exhibits the essential points of the more general result. Let $\phi$ be a 1.s.c. map from a separable metric space $X$ to the space of nonempty subsets of an Hilbert space $H$ ( $H$ need not be separable and it is taken in the $w$-topology). Assume that $\phi(x)$ is closed and convex for every $x \in X$ and that $\bigcup_{x \in X} \phi(x)$ is bounded. Then $\phi$ admits a continuous selection. This selection theorem is a consequence of a selection theorem of Michael and the fact (proved in [3]) that the space of the $w$-continuous functions from a separable metric space $X$ to a nonseparable Hilbert space is a Lindelöf space in the topology of pointwise convergence.

The selection theorems are stated and proved in the beginning of $\$ 2$. Next we show that the assumptions implying the existence of a continuous selection also imply that the continuous selections form a dense subset of the set of all Borel selections. (For a precise definition of this notion see the discussion preceding Theorem 2.4.) $\$ 2$ ends with some applications of our results. We indicate how 
some facts concerning linear operators between $C(K)$ spaces, which were proved (in [1], [12]) for compact metric $K$ by using Michael's theorems, can be extended to some nonmetrizable compact $K$ by applying the selection theorems proved here.

In $\$ 3$ we give examples which show the role of the various assumptions in the selection theorems of $\S 2$. One of the examples in $\S 3$ answers a problem raised by Michael [12].

Our notations and terminology are standard (taken from [6], [7]). We use also the notations given in the introduction of the preceding paper [3]. We recall that we consider linear spaces over the reals (this assumption is used only in the applications, Theorems 2.6, 2.7 and 2.8).

2. Selection theorem. We pass now to the statements and proofs of the selection theorems.

THEOREM 2.1. Let $\Gamma$ be a discrete space and let $X$ be a paracompact topological space such that every point $x$ in $X$ has a neighborhood which is a continuous image of a complete separable metric space (e.g. X may be any locally compact metric space). Let $\phi$ be a l.s.c. map from $X$ to $2^{C_{0}(\Gamma)}$ such that $\phi(x)$ is a compact convex (nonempty) subset of $C_{0}(\Gamma)$ for every $x \in X$. Then $\phi$ admits a continuous selection.

Proof. We assume first that $X$ itself is a continuous image of a complete separable metric space. Let $\left\{\Gamma_{\alpha}\right\}_{\alpha \in \Omega}$ denote the set of all countable subsets of $\Gamma$, and order $\Omega$ by inclusion (i.e., $\alpha>\beta$ iff $\Gamma_{\alpha} \supset \Gamma_{\beta}$ ). For every $\alpha \in \Omega$, let $P_{\alpha}$ be the canonical projection from $C_{0}(\Gamma)$ onto $C_{0}(\Gamma) / \Gamma_{\alpha}$, and put

$$
F_{\alpha}=\left\{f: f \in C_{p}\left(X, C_{0}(\Gamma)\right), P_{\alpha} f(x) \in P_{\alpha} \phi(x) \text { for every } x\right\} .
$$

Suppose we knew that $\bigcap_{\alpha} F_{\alpha} \neq \varnothing$. We claim that every member of this intersection is a continuous selection for $\phi$. Indeed, let $f \in \bigcap_{\alpha} F_{\alpha}$ and let $x_{0} \in X$. There is an $\alpha_{0} \in \Omega$ such that $P_{\alpha_{0}} f\left(x_{0}\right)=f\left(x_{0}\right)$. For every $\alpha>\alpha_{0}$ we have that $f\left(x_{0}\right)=P_{\alpha} f\left(x_{0}\right)=P_{\alpha} y_{\alpha}$ for some $y_{\alpha} \in \phi\left(x_{0}\right)$. Since $\phi\left(x_{0}\right)$ is compact the net $\left\{y_{\alpha}\right\}_{\alpha>\alpha_{0}}$ has a subnet converging to some $y_{0} \in \phi\left(x_{0}\right)$. It is clear that $y_{0}=f\left(x_{0}\right)$ and hence $f\left(x_{0}\right) \in \phi\left(x_{0}\right)$.

Thus we have only to prove that $\bigcap_{\alpha} F_{\alpha} \neq \varnothing$. Since $C_{p}\left(X, C_{0}(\Gamma)\right)$ is a Lindelöf space (Theorem 2.2 of [3]) and every $F_{\alpha}$ is closed, it is enough to show that $\bigcap_{i=1}^{\infty} F_{\alpha_{i}} \neq \varnothing$ for every sequence $\left\{\alpha_{i}\right\}_{i=1}^{\infty}$ in $\Omega$. Let $\alpha \in \Omega$ satisfy $\alpha>\alpha_{i}$ for every $i$. Then $P_{\alpha_{i}} P_{\alpha}=P_{\alpha_{i}}$ and hence $F_{\alpha} \subset \bigcap_{i=1}^{\infty} F_{\alpha_{i}}$. Therefore we have only to prove that $F_{\alpha} \neq \varnothing$ for every $\alpha$.

Let $\alpha \in \Omega$. We can clearly identify $C_{0}(\Gamma) / \Gamma_{\alpha}$ with $C_{0}\left(\Gamma_{\alpha}\right)$ and thus consider $C_{0}(\Gamma) / \Gamma_{\alpha}$ as a subset of $C_{p}\left(\Gamma_{\alpha}\right)$. The mapping $\phi_{\alpha}: X \rightarrow 2^{C_{p}\left(\Gamma_{a}\right)}$ defined by $\phi_{\alpha}(x)=P_{\alpha} \phi(x)$ is 1.s.c. and for every $x \in X, \phi_{\alpha}(x)$ is a (nonempty) convex and closed (even compact) subset of $C_{p}\left(\Gamma_{\alpha}\right)$. Since $X$ is paracompact and $C_{p}\left(\Gamma_{\alpha}\right)$ is a 
complete metrizable locally convex space, $\phi_{\alpha}$ admits a continuous selection $f$ by a theorem of Michael [10, Theorem 3.2"]. We have that, for every $x \in X$, $f(x) \in P_{\alpha} \phi(x) \subset C_{0}(\Gamma) / \Gamma_{\alpha} \subset C_{0}(\Gamma)$, and hence $P_{\alpha} f(x)=f(x) \in P_{\alpha} \phi(x)$. This concludes the proof if $X$ is a continuous image of a complete separable metric space.

Let now $X$ be a general space satisfying the assumption of the theorem. Then $X$ has a locally finite open covering by sets $\left\{G_{\beta}\right\}_{\beta \in B}$ such that each $G_{\beta}$ is a continuous image of a complete separable metric space. Let $\phi$ be a 1.s.c. map from $X$ to $2^{C_{0}(\Gamma)}$ such that $\phi(x)$ is convex compact for every $x$. By what we have already proved there is for every $\beta \in B$ a continuous function $f_{\beta}$ from $G_{\beta}$ to $C_{0}(\Gamma)$ such that $f_{\beta}(x) \in \phi(x)$ if $x \in G_{\beta}$. Let $\left(\psi_{\beta}\right\}_{\beta \in B}$ be a partition of unity subordinate to the covering $\left\{G_{\beta}\right\}_{\beta \in B}$. The function $f(x)=\Sigma_{\beta} \psi_{\beta}(x) f_{\beta}(x)$ is a continuous selection for $\phi$.

If, in Theorem 2.1, the range of $\phi$ is contained in a compact subset of $C_{0}(\Gamma)$ then Theorem 2.3 of [3] enables us to weaken somewhat the assumptions on $X$.

Theorem 2.2. Let $\Gamma$ be a discrete space and let $X$ be a paracompact topological space such that every $x \in X$ has a neighborhood which is a continuous image of a separable metric space. Let $\phi$ be a l.s.c. map from $X$ to $2^{C_{0}(\Gamma)}$ such that, for every $x \in X, \phi(x)$ is a compact convex set. Assume also that for every $x$ there is an open set $G_{x}$ containing $x$ such that $\overline{\bigcup_{z \in G . .} \phi(z)}$ is compact. Then $\phi$ admits a continuous selection.

Proof. As the last part of the proof of Theorem 2.1 shows, it is easy to pass from the local situation to the global one. Hence, without loss of generality, we may assume that $X$ itself is a continuous image of a separable metric space and that $L=\bigcup_{x \in X} \phi(x)$ is a compact subset of $C_{0}(\Gamma)$. Let $\left\{\Gamma_{\alpha}\right\}_{\alpha \in \Omega}$ and $P_{\alpha}$ be the same as in the proof of Theorem 2.1. Put $\tilde{L}=\bigcup_{\alpha \in \Omega} P_{\alpha} L$. $\tilde{L}$ is also a compact subset of $C_{0}(\Gamma)$. Indeed, let $\left\{P_{\alpha_{v}} y_{v}\right\}$ be a net in $\tilde{L}\left(y_{v} \in L\right.$ for every $\left.v\right)$. By the compactness of $L$ there is for every $\gamma \in \Gamma$ a number $\lambda_{\gamma}$ such that $|y(\gamma)|<\lambda_{\gamma}$ for every $y \in L$. Hence $L$ and therefore $\tilde{L}$ is contained in the compact space $\prod=\prod_{\gamma \in \Gamma}\left[-\lambda_{\gamma}, \lambda_{\gamma}\right]$. Let $\left\{P_{\alpha_{v^{\prime}}} y_{v^{\prime}}\right\}$ be a subnet of $\left\{P_{\alpha_{v}} y_{v}\right\}$ such that $y_{v^{\prime}}$ converges to some point $y \in L$ and $P_{\alpha_{v}}, y_{v}$, converges to some point $y_{0} \in \Pi$. For every $\gamma \in \Gamma$ we have that $y_{0}(\gamma)=0$ or $y_{0}(\gamma)=y(\gamma)$. Consequently $y_{0}=P_{\alpha_{0}} y$ for some $\alpha_{0} \in \Omega$ and hence $y_{0} \in \tilde{L}$.

We now proceed exactly as in the proof of Theorem 2.1 . We have only to replace the space $C_{p}\left(X, C_{0}(\Gamma)\right)$ used there by the space $C_{p}(X, L)$ which is a Lindelöf space by Theorem 2.3 of [3].

The following special case of Theorem 2.2 seems to be the most useful result for applications.

COROLlary 2.3. Let $X$ satisfy the same assumptions as in Theorem 2.2. Let $K$ be a w-compact set in the Banach space $L_{p}(\mu), 1 \leqq p<\infty$ ( $\mu$ an arbitrary measure), or a $w^{*}$-compact set in $L_{\infty}(\mu)$ (with $\mu \sigma$-finite). Then every l.s.c. map 
$\phi: X \rightarrow 2^{K}$ such that $\phi(x)$ is closed (nonempty) and convex for every $x$ admits a continuous selection ( $K$ is taken in the $w$ resp. $w^{*}$-topology).

Proof. Use Theorem 2.2 and the discussion following Theorem 2.3 of [3].

Our next aim is to state the selection theorems in a slightly stronger form. It is clear that once a selection theorem is proved it ensures in general not only the existence of one continuous selection but the existence of many such selections. In [10] and [11] Michael gave some results elaborating this point. Here we want to prove another result in this direction which, we feel, might be useful in applying the selection theorems. Our result can be stated briefly as follows: The continuous selections are dense in the Borel selections. In order to make this statement precise we have, of course, to specify the selection problem which we consider, the notion of a Borel selection, and in particular, the topology in which density is proved. There are many possibilities for choosing the setting and a suitable topology; we state here only one such result.

Let $X$ be a separable metric space and let $L=L_{p}(\mu)$ for some $1<p<\infty$ and some measure $\mu$. Let $S^{w}$ be the unit cell of $L$ in the $w$-topology. A function $g: X \rightarrow S^{w}$ is called a Borel function if $g^{-1}(V)$ is a Borel set in $X$ for every open subset $\bar{V}$ of $S^{w}$. Let $\rho$ be a finite regular Borel measure on $X$ and let $g: X \rightarrow S^{w}$ be a Borel function. The integral $\int_{X} g d \rho$ is the unique point $y \in L$ for which $y^{*}(y)=\int_{X} y^{*}(g(x)) d \rho(x)$ for every $y^{*}$ in $L^{*}$. Let $\phi: X \rightarrow 2^{s^{w}}$ be a 1.s.c. map such that $\phi(x)$ is a closed convex (nonempty) set for every $x \in X$. By $\mathscr{B}_{\phi}$ we denote the class of all Borel selections of $\phi$, i.e., all Borel functions $g: X \rightarrow S^{w}$ such that $g(x) \in \phi(x)$ for every $x$. The class of all continuous selections of $\phi$ will be denoted by $\mathscr{C}_{\phi}$.

With these notations we have

THEOREM 2.4. Let $T$ be the map from $\mathscr{B}_{\phi}$ to L defined by $T g=\int_{X} g d \rho$. Then $T \mathscr{C}_{\phi}$ is norm dense in $T \mathscr{B}_{\phi}$.

Proof. $T \mathscr{C}_{\phi}$ is clearly a convex subset of $T \mathscr{B}_{\phi}$ and therefore if $\overline{T \mathscr{C}_{\phi}} \neq \overline{T \mathscr{B}_{\phi}}$ there would exist a $y^{*} \in L^{*}$ with $\left\|y^{*}\right\|=1$, an $\varepsilon>0$ and a $g_{0} \in \mathscr{B}_{\phi}$ such that, for every $f \in \mathscr{C}_{\phi}$,

$$
\int_{X} y^{*}(f(x)) d \rho(x)+\varepsilon \leqq \int_{X} y^{*}\left(g_{0}(x)\right) d \rho(x) .
$$

The real-valued function $y^{*}\left(g_{0}(x)\right)$ is a bounded Borel function on $X$ and hence it is a uniform limit of simple Borel functions (i.e. Borel functions with a finite range). Let $\delta>0$ and put $V_{\delta}=\left\{y: y \in L,\left|y^{*}(y)\right|<\delta\right\}$. By the regularity of $\rho$ there are closed subsets $\left\{K_{i}\right\}_{i=1}^{n}$ in $X$ and points $\left\{y_{i}\right\}_{i=1}^{n}$ in $S^{w}$ such that

$$
K_{i} \cap K_{j}=\varnothing \quad \text { if } i \neq j,
$$$$
g_{0}(x)-y_{i} \in V_{\delta} \quad \text { if } x \in K_{i} \text {, }
$$$$
|\rho|\left(X \sim \bigcup_{i=1}^{n} K_{i}\right)<\delta
$$ 
$(|\rho|(A)$ denotes the variation of $\rho$ on the set $A)$. We define now $\phi_{0}: X \rightarrow 2^{S^{w}}$ by

$$
\phi_{0}(x)= \begin{cases}\overline{\phi(x) \cap\left(y_{i}+V_{\delta}\right)} & \text { if } x \in K_{i}, \\ \phi(x) & \text { if } x \in X \sim \bigcup_{i=1}^{n} K_{i} .\end{cases}
$$

It is easily checked that $\phi_{0}$ is 1.s.c. and that every $\phi_{0}(x)$ is closed convex and nonempty. By Corollary $2.3 \phi_{0}$ admits a continuous selection, $f$ say. Clearly $f \in \mathscr{C}_{\phi}$ and

$$
\begin{aligned}
\mid \int_{X} y^{*}(f(x) & \left.-g_{0}(x)\right) d \rho(x) \mid \\
\leqq & \sum_{i=1}^{n} \int_{K_{i}}\left|y^{*}\left(f(x)-g_{0}(x)\right)\right| d|\rho|(x) \\
& \quad+\int_{X \sim K_{i}}\left(\left|y^{*}\left(g_{0}(x)\right)\right|+\left|y^{*}(f(x))\right|\right) d|\rho|(x) \\
\leqq & \sum_{i=1}^{n} 2 \delta|\rho|\left(K_{i}\right)+2|\rho|\left(X \sim \cup K_{i}\right) \leqq 2 \delta\|\rho\|+2 \delta
\end{aligned}
$$

and this contradicts (2.1) if $\delta<\varepsilon / 2(\|\rho\|+1)$.

For the application of our selection theorems we need the following lemma which is proved in [4].

LEMMA 2.5. Let $K$ be a compact Hausdorff space. Then the unit cell of $C(K)^{*}$ in the $w^{*}$-topology is affinely homeomorphic to a subset of $C_{0}(\Gamma)$ for some discrete $\Gamma$ if and only if $K$ is homeomorphic to a subset of $C_{0}(\Gamma)$ for some discrete $\Gamma$.

It follows from Theorem 2.2 and Lemma 2.5 that the following stronger version of a result of Michael [12] is true.

THEOREM 2.6. Let $K_{1}$ be a compact subset of $C_{0}(\Gamma)$ for some discrete space $\Gamma$ and let $K_{2}$ be a compact metric space. Assume that there is a continuous open map $\psi$ from $K_{1}$ onto $K_{2}$. Then there exists a linear operator $T$ from $C\left(K_{1}\right)$ into $C\left(K_{2}\right)$ such that

$$
\inf f\left[\psi^{-1}(k)\right] \leqq T f(k) \leqq \sup f\left[\psi^{-1}(k)\right]
$$

for every $k \in K_{2}$ and $f \in C\left(K_{1}\right)$.

Michael [12] proved this result under the assumption that $K_{1}$ is compact metric. The proof given in [12] works also in the present, more general, situation if Theorem 2.2 is used instead of Michael's selection theorem. We shall show 
in the next section that Theorem 2.6 may fail to hold if $K_{1}$ is a general compact Hausdorff space.

The theorems of this section can also be used for obtaining some additional information concerning the main result of [1]. We present here two results in this direction. The proofs are very similar to that of Theorem 1 of [1]. We omit the details.

Proposition 2.7. Let $K_{1}$ be a compact subset of $C_{0}(\Gamma)$ for some discrete space $\Gamma$ and let $K_{2}$ be a compact metric space. Denote by $S\left(K_{1}, K_{2}\right)$ the set of all linear operators $T$ from $C\left(K_{1}\right)$ to $C\left(K_{2}\right)$ with $\|T\| \leqq 1$. Let $T_{0} \in S\left(K_{1}, K_{2}\right)$. Then $T_{0}$ is an extreme point of $S\left(K_{1}, K_{2}\right)$ if and only if $T_{0}$ is of the form

$$
T_{0} f(k)=\lambda(k) f(\psi(k)), \quad k \in K_{2}, \quad f \in C\left(K_{1}\right)
$$

where $\psi \in C\left(K_{2}, K_{1}\right)$ and $\lambda \in C\left(K_{2}\right)$ with $|\lambda(k)|=1$ for every $k \in K_{2}$.

Proposition 2.8. Let $K_{1}$ and $K_{2}$ be compact Hausdorff spaces, and assume that $K_{2}$ is separable (i.e. has a dense countable subset). Denote by $W\left(K_{1}, K_{2}\right)$ the set of all linear weakly compact operators $T$ from $C\left(K_{1}\right)$ to $C\left(K_{2}\right)$ with $\left\|T_{:}\right\| \leqq 1$. Let $T_{0} \in W\left(K_{1}, K_{2}\right)$. Then $T_{0}$ is an extreme point of $W\left(K_{1}, K_{2}\right)$ if and only if $T_{0}$ is of the form (2.3).

REMARK. It is easily seen that every $w$-compact $T_{0}$ of the form (2.3) is compact.

It is likely that Propositions 2.7 and 2.8 hold for all compact Hausdorff $K_{1}$ and $K_{2}$. Proposition 2.8 can be proved, for metrizable $K_{2}$, by using Theorem 2.2 $\left(C\left(K_{1}\right)^{*}\right.$ is $L_{1}(\mu)$ for some measure $\left.\mu\right)$. However, it is easy to reduce the problem to a selection problem with a metrizable range, and so the selection theorem of Michael suffices for this purpose.

3. Examples. The purpose of the first half of this section is to examine the necessity of the various assumptions in Theorems 2.1 and 2.2.

(1) Theorem 2.1 does no longer hold (even for countable $\Gamma$ ) if we weaken the assumption that $\phi(x)$ is compact convex for every $x$ by assuming only that $\phi(x)$ is closed and convex. To see this, take as $\Gamma$ the integers $N$ and let $X=[0,1]$. Order the rational points in $X$ into a sequence $r_{n}$ and put

$$
\phi(x)= \begin{cases}\left\{y: y \in C_{0}(N),|y(i)| \leqq 1, i=1,2, \cdots\right\} & \text { if } x \text { is irrational, } \\ \left\{y: y \in C_{0}(N),|y(i)| \leqq 1, i=1,2, \cdots, y(n)=\frac{1}{2}\right\} & \text { if } x=r_{n} .\end{cases}
$$

It is easily checked that $\phi$ is l.s.c., and that $\phi(x)$ is closed convex and bounded for every $x$. $\phi$ does not admit a continuous selection. Indeed, assume that $f: X \rightarrow C_{0}(N)$ is continuous and that $f(x) \in \phi(x)$ for every $x$. Then there is an open interval $I_{1}$ around $r_{1}$ such that $|f(x)(1)| \geqq 1 / 4$ if $x \in \bar{I}_{1}$. Let $n_{2}>n_{1}=1$ be such that $r_{n_{2}} \in I_{1}$. There is an open interval $I_{2} \subset I_{1}$ around $r_{n_{2}}$ such that 
$\left|f(x)\left(n_{2}\right)\right| \geqq 1 / 4$ if $x \in \bar{I}_{2}$. Continuing in this manner we get a sequence of integers $1=n_{1}<n_{2}<n_{3}<\cdots$ and a sequence of intervals $I_{1} \supset I_{2} \supset I_{3} \cdots$ such that $\left|f(x)\left(n_{k}\right)\right| \geqq 1 / 4$ if $x \in I_{k}$. Let $x_{0} \in \bigcap_{k=1}^{\infty} I_{k}$ then $f\left(x_{0}\right)$ has an infinite number of coordinates $\geqq 1 / 4$ and this contradicts the assumption that $f\left(x_{0}\right) \in C_{0}(N)$. This example is a variant of Example 6.2 of Michael [10].

We shall now give examples which show that if we consider 1.s.c. mappings $\phi$ into the compact convex subsets of a Banach space in the $w$-topology, there may be no selection for $\phi$ if $\bigcup_{x} \phi(x)$ is not contained in a $w$-compact set. In, fact even if $X=N^{*}$, the one point compactification of the integers, and $\phi(x)$ is norm compact and convex for every $x$, Corollary 2.3 may fail to hold if $K$ is not $w$ compact:

(2) Let us consider the map $\phi: N^{*} \rightarrow 2^{l_{1}}$ defined by

$$
\phi(n)=\left\{y: y=\left(y_{1}, y_{2}, \cdots, y_{n}, 1 / 2,0,0, \cdots\right), \sum_{i=1}^{n}\left|y_{i}\right| \leqq 1 / 2\right\}
$$

if $n \in N$ and $\phi(\infty)=\{0\}$ ( $\infty$ denotes the point in $N^{*} \sim N$ ). In $l_{1}$ a sequence converges $w$ to 0 iff it converges in norm to 0 . Hence $\phi$ does not have a continuous selection. Clearly $\phi(n)$ is convex and norm compact for every $n$. Let us verify that $\phi$ is $1 . s . c$. if we take in $l_{1}$ the $w$-topology. Let $u^{j}=\left(u_{1}^{j}, u_{2}^{j}, \cdots\right), j=1, \cdots, k$ be elements in $l_{\infty}$. We have to show that there exists an integer $n_{0}$ such that for $n>n_{0}$ there is a $y \in \phi(n)$ with $\left|\left(u^{j}, y\right)\right|=\left|\Sigma_{i}^{j} u_{i} y_{i}\right| \leqq 1$ for every $j$. Denote by $\psi(n)$ the set $\left\{y: y=\left(y_{1}, y_{2}, \cdots, y_{n}, 0,0, \cdots\right), \sum_{i=1}^{n}\left|y_{i}\right| \leqq 1 / 2\right\}$, and let $T: l_{1} \rightarrow R^{k}$ be defined by $T y(j)=\left(u^{j}, y\right), j=1, \cdots, k$. Since $T \psi\left(n_{1}\right) \subset T \psi\left(n_{2}\right)$ if $n_{1}<n_{2}$, it follows by the compactness of $\overline{\bigcup_{n} T \psi(n)}$ that there is an $n_{0}$ such that, for every $n>n_{0}$ and $y \in \psi(n)$, there is $y_{0} \in \psi\left(n_{0}\right)$ such that

$$
\left|\left(u^{j}, y_{0}\right)-\left(u^{j}, y\right)\right| \leqq 1, \quad j=1,2, \cdots, k .
$$

Let now $n>n_{0}$ and take $y=(0,0, \cdots, 0,1 / 2,0, \cdots)(1 / 2$ in the $(n+1)$ th place and all other coordinates are 0$)$ and let $y_{0} \in \psi\left(n_{0}\right)$ satisfy (3.1). Then $\tilde{y}=y-y^{0} \in \phi(n)$ and $\left|\left(u^{j}, \tilde{y}\right)\right| \leqq 1$ for every $j$.

(3) In the example just described, all the $\phi(n)$ are contained in the unit cell of $l_{1}$. If $1<p<\infty$; then every $w$-closed and bounded subset of $l_{p}$ is $w$-compact. Consequently, in order to construct a 1.s.c. $\phi: N^{*} \rightarrow 2^{l_{p}}$, with $\phi(n)$ compact convex for every $n$, which does not admit a continuous selection, $\bigcup_{n=1}^{\infty} \phi(n)$ must be unbounded. Take, for example, $p=2$ and define $\phi: N^{*} \rightarrow 2^{l_{2}}$ by

$$
\phi(n)=\left\{y: y=\left(y_{1}, y_{2}, \cdots, y_{n}, n, 0,0, \cdots\right), \sum_{i=1}^{n}\left|y_{i}\right|^{2} \leqq n^{2}\right\}
$$

and let $\phi(\infty)=\{0\}$. If we take the $w$-topology in $l_{2}$, then $\phi$ is 1.s.c. (cf. Lemma 3.1 below) but $\phi$ does not have a continuous selection. 
We turn now to selections with range in a $w^{*}$-compact set of a conjugate Banach space. The mapping $\phi: N^{*} \rightarrow 2^{l_{1}}$ of example (2) can also be considered as a mapping $\tilde{\phi}: N^{*} \rightarrow 2^{l_{1}{ }^{* *}}=2^{l_{\infty}^{*}}$ (we embed $l_{1}$ canonically in $l_{1}^{* *}$ ). The mapping $\tilde{\phi}$ is 1 .s.c. (we take in $l_{\infty}^{*}$ the $w^{*}$-topology), $\tilde{\phi}(x)$ is closed and convex for every $x \in N^{*}$, and $\bigcup_{n} \tilde{\phi}(n)$ is contained in the $w^{*}$-compact unit cell of $l_{\infty}^{*}$. Since $\tilde{\phi}$ does not have a continuous selection we see that Corollary 2.3 does not hold if $K$ is an arbitrary $w^{*}$-compact set. Actually, the corollary does not hold even for $K$ the unit cell of $\left(L_{1}(\mu)\right)^{*}$ in the $w^{*}$-topology if $\mu$ is not $\sigma$-finite (thus the $\sigma$-finiteness assumption in the statement of the corollary cannot be discarded). In fact, every $w^{*}$-compact set can be embedded (affinely and topologically) in the unit cell of $l_{\infty}(\Gamma)=l_{1}(\Gamma)^{*}$ if $\Gamma$ is a discrete set of a sufficiently large cardinality.

We now consider the question of the necessity of the assumptions on $X$ in Theorems 2.1 and 2.2. We do not know whether the completeness assumption in Theorem 2.1 is necessary. The assumption of the local separability of $X$ in Theorems 2.1 and 2.2 is essential as the following example shows.

(4) Let $H$ be a Hilbert space having an orthonormal basis of cardinality $\aleph_{1}$. We denote a fixed basis of $H$ by $\left\{e_{\alpha}\right\}$, where $\alpha$ ranges over all the ordinals less than the first uncountable ordinal $\Omega$. Let $X$ be the subspace of $H$ consisting of the points $e_{\alpha} / n(\alpha<\Omega, n=1,2, \cdots)$ and 0 . We take in $X$ the metric induced on it by the norm of $H$. $X$ is a complete metric space and 0 is the only nonisolated point in it. Let $S^{w}$ be the unit cell of $H$ in the $w$-topology. For every infinite ordinal $\alpha<\Omega$ we order the set $\{\beta: \beta<\alpha\}$ into a sequence and denote this sequence by $\beta_{1, \alpha}, \beta_{2, \alpha} \cdots$.

Define $\phi: X \rightarrow 2^{S^{w}}$ by

$$
\phi(x)= \begin{cases}\{0\} & \text { if } x=0 \text { or } x=e_{\alpha} / n \text { with } \alpha \text { a finite ordinal, } \\ {\left[e_{\alpha}, e_{\beta_{n, \alpha}}\right]} & \text { if } x=e_{\alpha} / n \text { with } \alpha \text { an infinite ordinal, }\end{cases}
$$

where $[u, v]$ denotes the closed segment joining the points $u$ and $v$.

We show that $\phi$ is lower semicontinuous, i.e., that for every $w$-neighborhood $V$ of 0 in $S^{w}$ there is an $n_{0}$ such that $\phi\left(e_{\alpha} / n\right) \cap V \neq \varnothing$ for every $\alpha<\Omega$ and every $n>n_{0}$. Let $\alpha_{1}, \alpha_{2}, \cdots, \alpha_{k}$ be ordinals $<\Omega$ and let $n_{0}$ be an integer such that $\alpha_{i}=\beta_{m, \alpha_{j}}$ with $m<n_{0}$ whenever $\alpha_{i}<\alpha_{j}(1 \leqq i, j \leqq k)$ and $\alpha_{j}$ is infinite. Let $\alpha$ be an infinite ordinal $<\Omega$ and let $n>n_{0}$. If $\alpha \neq \alpha_{j}, j=1, \cdots, k$ then

$$
e_{\alpha} \in \phi\left(e_{\alpha} / n\right) \cap\left\{u: u \in S^{w},\left(u, e_{\alpha_{j}}\right)=0, j=1, \cdots, k\right\} .
$$

If $\alpha=\alpha_{j}$ for some $j \leqq k$ then by the choice of $n_{0}$

$$
e_{\beta_{n, \alpha}} \in \phi\left(e_{\alpha} / n\right) \cap\left\{u: u \in S^{w},\left(u, e_{\alpha_{j}}\right)=0, j=1, \cdots, k\right\} .
$$

This shows that $\phi$ is 1.s.c.

The map $\phi$ does not admit a continuous selection. Indeed, assume that 
$f: X \rightarrow S^{w}$ is a continuous function such that, for infinite $\alpha, f\left(e_{\alpha} / n\right)$ $=\lambda(\alpha, n) e_{\alpha}+(1-\lambda(\alpha, n)) e_{\beta_{n}, \alpha}$ with $0 \leqq \lambda(\alpha, n) \leqq 1$, and $f(0)=0$. Then for every $\gamma<\Omega$ there is an $n_{\gamma}$ such that for $n>n_{\gamma}$ and every $\alpha<\Omega,\left(f\left(e_{\alpha} / n\right), e_{\gamma}\right)<1 / 3$. There is an $n_{0}$ and an uncountable set $\Gamma \subset\{\alpha: \alpha<\Omega\}$ such that $n_{\gamma}=n_{0}$ for every $\gamma \in \Gamma$. Let $\gamma_{0} \in \Gamma$ be such that $\Gamma \cap\left\{\alpha: \alpha<\gamma_{0}\right\}$ is infinite. Then there is a $\gamma_{1} \in \Gamma$ such that $\gamma_{1}=\beta_{n, \gamma_{0}}$ with $n>n_{0}$. Hence

$$
\lambda\left(\gamma_{0}, n\right)=\left(f\left(e_{\gamma_{0}} / n\right), e_{\gamma_{0}}\right)<1 / 3
$$

and

$$
1-\lambda\left(\gamma_{0}, n\right)=\left(f\left(e_{\gamma_{0}} / n\right), e_{\gamma_{1}}\right)<1 / 3,
$$

and this is a contradiction.

Since every metric space which is not locally separable has a closed subset homeomorphic to the space $X$ of example (4), it follows that Theorem 2.2(and Corollary 2.3) hold for a metric space $X$ if and only if $X$ is locally separable.

There are examples [8], [9], [2] of compact Hausdorff spaces $X$ for which Theorems 2.1 and 2.2 do not hold. These examples show that even the extension theorem which corresponds to (i.e. is a special case of) Theorem 2.1 may fail to hold. In the examples $X$ may be taken to be even separable or a $w$-compact set in a Banach space (but of course not both $w$-compact and separable). We refer to [2] for details.

We conclude the paper with an example which shows that Theorem 2.6 may fail to hold if $K_{1}$ is an arbitrary compact Hausdorff space. This solves a problem raised by Michael [12].

(5) Let $H^{w}$ be the separable Hilbert space $l_{2}$ with the $w$-topology, and let $\beta\left(H^{w}\right)$ be its Stone-Čech compactification. For every $n \in N$ let $A_{n}$ be the subset of $H^{w}$ defined by

$$
A_{n}=\left\{y: y=\left(y_{1}, y_{2}, \cdots, y_{n}, n, 0, \cdots\right), \sum_{i=1}^{n}\left|y_{i}\right|^{2} \leqq n^{2}\right\} .
$$

Each $A_{n}$ is compact (even norm compact) and hence it is a closed subset of $\beta\left(H^{w}\right)$. Let $K$ be the subset of $N^{*} \times \beta\left(H^{w}\right)$ defined by

$$
K=\bigcup_{n=1}^{\infty}\left(\{n\} \times A_{n}\right) \cup\left(\{\infty\} \times \beta\left(H^{w}\right)\right) .
$$

$K$ is a closed subset of $N^{*} \times \beta\left(H^{w}\right)$ and hence it is compact Hausdorff. Let $\psi$ be the map from $K$ to $N^{*}$ defined by $\psi(\gamma, y)=\gamma, \gamma \in N^{*}, y \in \beta\left(H^{w}\right)$. Clearly $\psi$ is continuous and onto. That $\psi$ is open follows from

LEMMA 3.1. Let $V$ be an open subset of $H^{w}$. Then there is an $n_{0}$ such that for $n>n_{0}, A_{n} \cap V \neq \varnothing$.

Proof. $V$ contains a set of the form $\left\{y:\left|\left(y-z, u^{j}\right)\right|<1, j=1, \cdots, m\right\}$ for 
some $z$ and $\left\{u^{j}\right\}_{j=1}^{m}$ in $H^{w}$. We assume, as we may, that the $u^{j}=\left(u_{1}^{j}, u_{2}^{j}, \cdots\right)$ are linearly independent. Hence there exist integers $k_{1}<k_{2}<\cdots<k_{m}$ such that the $m \times m$ matrix $M=\left(u_{k_{i}}^{j}\right)_{1 \leqq i}, j \leqq m$ is nonsingular. Let $n>k_{m}$ and consider the system of equations

$$
\sum_{i=1}^{m} \alpha_{i} u_{k_{i}}^{j}=-u_{n}^{j}+\left(u^{j}, z\right) / n, \quad j=1, \cdots, m .
$$

Since $M$ is nonsingular this system has a unique solution $\left(\alpha_{1}(n), \alpha_{2}(n), \cdots, \alpha_{m}(n)\right)$ given by

$$
\left[\begin{array}{c}
\alpha_{1}(n) \\
\alpha_{2}(n) \\
\vdots \\
\alpha_{m}(n)
\end{array}\right]=M^{-1}\left[\begin{array}{c}
-u_{n}^{1}+\left(u^{1}, z\right) / n \\
-u_{n}^{2}+\left(u^{2}, z\right) / n \\
\vdots \\
-u_{n}^{m}+\left(u^{m}, z\right) / n
\end{array}\right] .
$$

Since for every $j, u_{n}^{j} \rightarrow 0$ and $\left(u^{j}, z\right) / n \rightarrow 0$ as $n \rightarrow \infty$ there is an $n_{0}$ such that, for $n>n_{0}, \sum_{i=1}^{m} \alpha_{i}(n) \leqq 1$. Hence for $n>n_{0}$ the vector

$$
y^{n}=\left(0, \cdots, 0, n \alpha_{1}(n), 0, \cdots, 0, n \alpha_{2}(n), 0, \cdots, 0, n \alpha_{m}(n), 0, \cdots, 0, n, 0,0 \cdots\right)
$$

$\left(n \alpha_{i}(n)\right.$ stands at the $k_{i}$ th place and $n$ at the $(n+1)$ th place) belongs to $A_{n}$. Also

$$
\left(u^{j}, y^{n}\right)=\sum_{i=1}^{m} n \alpha_{i}(n) u_{k_{i}}^{j}+n u_{n}^{j}=\left(u^{j}, z\right)
$$

for every $j$, and hence $y^{n} \in V$. This concludes the proof of Lemma 3.1 and hence of the fact that $\psi$ is open.

We shall now prove that there does not exist a linear operator $T$ from $C(K)$ to $C\left(N^{*}\right)$ for which $(2.2)$ holds. This assertion is easily seen to be equivalent to the following:

(a) There does not exist a $w^{*}$-convergent sequence of measures $\mu_{1}, \mu_{2}, \cdots$ on $K$ such that $\mu_{n} \geqq 0, \mu_{n}\left(\{n\} \times A_{n}\right)=1$ and $\mu_{n}\left(K \sim\{n\} \times A_{n}\right)=0$ for every $n$.

We shall show here that the following stronger assertion is valid.

(b) There does not exist a $w^{*}$-convergent sequence of finite measures $\mu_{1}, \mu_{2}, \cdots$ on $\beta\left(H^{w}\right)$ such that $\mu_{n}\left(A_{n}\right) \geqq 1$ and such that the support of $\mu_{n}$ is contained in $A_{n}$ for every $n$.

That (b) $\Rightarrow$ (a) follows from the fact that if $f \in C\left(\beta\left(H^{w}\right)\right)$ then the function $f$ is an element of $C\left(N^{*} \times \beta\left(H^{w}\right)\right)$ where $f(\gamma, y)=f(y), \gamma \in N^{*}, y \in \beta\left(H^{w}\right)$.

For the proof of (b) we shall need the following, certainly essentially known, lemma.

LEMMA 3.2. Let $\left\{\mu_{n}\right\}_{n=1}^{\infty}$ be a sequence of finite measures on $[-1,1]$. Assume that for every bounded and continuous function $g$ from $R$ into itself

$$
\lim _{n \rightarrow \infty} \int_{-1}^{1} g(n t) d \mu_{n}(t)
$$


exists. Then for every $\varepsilon>0$

$$
\lim _{n \rightarrow \infty}\left|\mu_{n}\right|(\{t:|t|>\varepsilon\})=0 .
$$

$(|\mu|(B)$ denotes the total variation of $\mu$ on the set $B$.

Proof. Assume there is a $\delta>0$ and a sequence $n_{1}<n_{2}<n_{3} \cdots$ of integers such that $\left|\mu_{n_{k}}\right|(\{t:|t|>\varepsilon\})>\delta$. We may assume that $\varepsilon n_{k+1}>n_{k}$ for every $k$. Let $\theta_{k} \subset C(R)$ satisfy $\left|\theta_{k}(t)\right| \leqq 1$ for every $t, \theta_{k}(t) \neq 0 \Rightarrow \varepsilon n_{k}<|t|<n_{k}$, and $\int_{-1}^{1} \theta_{k}\left(n_{k} t\right) d \mu_{n_{k}}(t)>\delta / 2$. Let $g(t)=\sum_{k=1}^{\infty} \varepsilon_{k} \theta_{k}(t)$ with $\varepsilon_{k}= \pm 1$ where the signs $\varepsilon_{k}$ are chosen inductively so that

$$
\left|\int_{-1}^{1} g\left(n_{k} t\right) d \mu_{n_{k}}(t)-\int_{-1}^{1} g\left(n_{k-1} t\right) d \mu_{n_{k-1}}(t)\right|>\delta / 2 .
$$

This is possible since changing the sign $\varepsilon_{k}$ does not change the value of

$$
\int_{-1}^{1} g\left(n_{k-1} t\right) d \mu_{n_{k-1}}(t)
$$

while

$$
\int_{-1}^{1} g\left(n_{k} t\right) d \mu_{n_{k}}(t)
$$

is changed by at least $\delta$. But (3.2) contradicts our assumption and thus we have proved the lemma.

We pass to the proof of statement (b). Suppose (b) were false. In view of the definition of $A_{n}$ there would be, for every $n$, a measure $v_{n}$ on the set

$$
S_{n}=\left\{y: y \in H^{w}, y=\left(y_{1}, y_{2}, \cdots, y_{n}, 0,0,0, \cdots\right),\|y\| \leqq 1\right\}
$$

with $v_{n}\left(S_{n}\right) \geqq 1$, such that for every bounded $f$ in $C\left(H^{w}\right)$ the following limit

$$
\lim _{n \rightarrow \infty} \int_{S_{n}} f\left(n y_{1}, n y_{2}, \cdots, n y_{n}, n, 0,0, \cdots\right) d v_{n}(y)
$$

exists. Let $u=\left(u_{1}, u_{2}, \cdots, u_{k}, 0,0 \cdots\right)$ be any point in $H^{w}$ with only a finite number of nonzero coordinates. We claim that for every $\varepsilon>0$

$$
\lim _{n \rightarrow \infty}\left|v_{n}\right|\left(\left\{y: y \in S_{n},|(u, y)|>\varepsilon\right\}\right)=0
$$

Indeed, let $g(t)$ be any bounded function in $C(R)$. Then $f(y)=g((u, y))$ is a bounded function in $C\left(H^{w}\right)$. Hence, by (3.3),

$$
\lim _{n \rightarrow \infty} \int_{S_{n}} g\left(n \sum_{i=1}^{k} u_{i} y_{i}\right) d v_{n}(y)
$$

exists. Consider now the measures $\rho_{n}$ on $I=[-\|u\|,\|u\|]$ defined by $\rho_{n}(A)$ 
$=v_{n}\left(\left\{y: y \in S_{n},(u, y) \in A\right\}\right)$ for every Borel subset $A$ of $I$. By applying Lemma 3.2 to the measures $\rho_{n}$ we see that (3.5) implies (3.4).

It follows from (3.4) that for every $\varepsilon>0$

$$
\lim _{n \rightarrow \infty}\left(\int_{S_{n}} \operatorname{arctg}(n(u, y)+n \varepsilon) d v_{n}(y)-\pi v_{n}\left(S_{n}\right) / 2\right)=0
$$

and

$$
\lim _{n \rightarrow \infty}\left(\int_{S_{n}} \operatorname{arctg}(n(u, y)-n \varepsilon) d v_{n}(y)+\pi v_{n}\left(S_{n}\right) / 2\right)=0 .
$$

By (3.6), (3.7) and an easy inductive argument it follows that there is a sequence $n_{0}<n_{1}<n_{2} \cdots$ of integers such that if $\tilde{u}$ is the point in $H^{w}$ defined by

$$
\tilde{u}_{i}= \begin{cases}(-1)^{k} / 2^{k+1} & \text { if } i=n_{k}+1, k=0,1 \cdots, \\ 0 & \text { for all other } i,\end{cases}
$$

then

$$
\int_{S_{n_{k}}} \operatorname{arctg}\left(n_{k} \sum_{i=1}^{n_{k}} \tilde{u}_{i} y_{i}+n_{k} \tilde{u}_{n_{k}+1}\right) d v_{n_{k}}(y) \quad \begin{cases}\geqq \pi / 4, & k \text { even, } \\ \leqq-\pi / 4, & k \text { odd }\end{cases}
$$

But (3.8) contradicts (3.2) for the function $f$ defined by $f(y)=\operatorname{arctg}(u, y)$. This concludes the proof of assertion (b) and thus of the nonexistence of an operator $T$ satisfying (2.2).

\section{REFERENCES}

1. R. M. Blumenthal, J. Lindenstrauss and R. R. Phelps, Extreme operators into $C(k)$, Pacific J. Math. (to appear).

2. H. H. Corson and J. Lindenstrauss, On simultaneous extension of continuous functions,

3. - On function spaces which are Lindelöf spaces, Trans. Amer. Math. Soc. 121 (1966), 476-491.

4. - On weakly compact subsets of Banach spaces, Proc. Amer. Math. Soc. (to appear).

5. J. Dugundji, An extension of Tietze's theorem, Pacific J. Math. 1 (1951), 353-367.

6. N. Dunford and J. Schwartz, Linear operators, Part I, Interscience, New York, 1958.

7. J. L. Kelley, General topology, Van Nostrand, Princeton, N. J., 1955.

8. V. Klee, Convex bodies and periodic homoemorphisms in Hilbert space, Trans. Amer. Math. Soc. 74 (1953), 10-63.

9. E. Michael, Some extension theorems for continuous functions, Pacific J. Math. 3 (1953), 789-806.

10. —- Continuous selections. I, Ann. of Math. 63 (1956), 361-382.

11. —- Dense families of continuous selections, Fund. Math. 47 (1959), 173-178.

12. - A linear mapping between function spaces, Proc. Amer. Math. Soc. 15 (1964), $415-416$.

UNIVERSITY OF WASHINGTON, SeatTle, Washington 